\title{
Airline Mobile Reservation Development
}

\author{
Dr. Mohammed Najm Abdullah ${ }^{1}$, Emtinan Haider Kadhim ${ }^{2}$ \\ Computer Engineering Department, UOT, Iraq ${ }^{1}$ \\ UOICT, Iraq $^{2}$
}

\begin{abstract}
As the technology innovations are quickly developed including mobile technology, mobile application for reservation services can enhance individuals' life, make it less complex. This research presents a mobile reservation system for airline companies to simplify the process of booking a flight. The users usually buy their ticket manually and maybe wait in a queue to check the required flight. The proposed system implemented to overcome manual system problem by making booking from home or elsewhere and save time and effort.
\end{abstract}

Keywords: ARS, mobile application, reservation system, Android, airline, booking.

\section{INTRODUCTION}

The most technological growth in the world have been within the last years, the improvements in different field made life easier, less complicated and extra comfy. One of these improvements is airline industry which has developed into one of the most advanced industries these days. Nowadays thousands of flights are needed by individuals daily. Thus the economies of countries also related individuals and societies cultures will be reinforced. The improvement of innovation has brought about huge advancement in flight booking system through last years. The manual ticket systems on the beginning of airline travels were just from paper and were to be gathered from the travel organizations or Airline Company in the wake of buying. With the globalization and the progress of airline industry, the procedure of reserving flight and buying ticket has additionally changed. Since the fast development and utilization of internet and mobile technology, mobile applications for reservation services become more common and make life easier. This led to reduce the utilization of manual system mechanism thatspent more time and efforts. Airline companies who deal with mobile reservation system all information of users include the data of the traveller's name, flight date, the flight number, flight destination, wages, ticket legality, condition if there is any change in reservation and refunds, would be save automatically and safety, that make reservation process easiest and organized.

\section{LITERATURE SURVEY}

Many researchers have applied many algorithms in online reservation system these work can be presented as following:

Oyelade 0. J. et al. in (2009) [3], Olaniyi, et al. in (2010) [4], Pranjali kharwade et al. in (2014) [6]presented a mobile application for airline reservation system with some differences in design methodology. Oyelade proposed system was produced utilizing the Wireless Markup Language (WML) as frontend, MySQL 4.0 database administration framework as back-end and PHP (Hypertext Preprocessor) as the server-side scripting language, while Olaniyi depended on .NET framework and MY SQL 2005 database management system for the back-end. And Pranjali introduced android application for ticket reservation and validation using mobile tower network. The system used SQL lite database and served as medium for people to book a ticket to travel through metros or locals.

Ahmed K. Ibrahim and Azman B Ta'a[7] and Subarnarekha Ghosal et al. [10] in 2015, developed android application for ticket booking. Ahmed developed a prototype of mobile bus ticketing system (MBTS) in Iraq using agile software development approach and Unified Modeling Language (UML) using Phonegap framework. Also JavaScript, jQuery mobile, HTML5, and CSS3 on mobile side also applied. Besides that, MySQL Database and PHP on the server side with RESTful used to serve a mobile application request. While Ghosal presented Android Suburban Ticket (ASR), A CLOUD database is used to store user data for security purpose, java language used to implement the system with PHP development framework.

Pooja Gautam, in 2015[8] and Chintan Shah, Wenbin Luo in 2016 [11]: Introduced a dynamic web application for reservation system, Pooja's airline system working with databases SQL lite, programming languages Python and JavaScript as well as using local server in Django to run the application. Chintan's workshop reservation system used an Apache web server hosting that understands PHP scripting language and is connected to a MySQL database, which decreased the amount of paperwork, time, and effort which spent by an attendee on registration operation. It can also reduce the errors resulted of the manual registration process.

\section{III.PROBLEM STATEMENT}

Since the manual system is slow, consume in time, boring and it is troublesome for every individual to book through office operators, collect information about client that they got through telephones, problem in update passenger information if any changes released. With the 


\section{International Advanced Research Journal in Science, Engineering and Technology \\ ISO 3297:2007 Certified}

Vol. 3, Issue 10, October 2016

development of information technology and use it in an orderly and properly helps to overcome the existing problems in the manual system. Booking system saves the customer information in a database, and this makes it easier in a way to save reservation information. Customer can book and reserve and buy tickets accordingly with save time and effort for the user and the airline employee. Also the users' data are saved simply and accurately the client never loses his ticket as in the manual system.

\section{IV.PROPOSED SYSTEM}

The created system will ease airline booking create account to the client, save details about client, gives menu of trips and their costs and dates to be reserved and there is a customer care services and details about the company and how to contact them. Figure 1 flow chart of system working.

\section{A. System design}

The framework based mobile reservation application uses client/server architecture. At The client by using android device can connect via internet with server that PHP and MySQL in server side responsible for the users' requests processes and save or return data from database.

Figure 1 represents overview of proposed reservation system design.

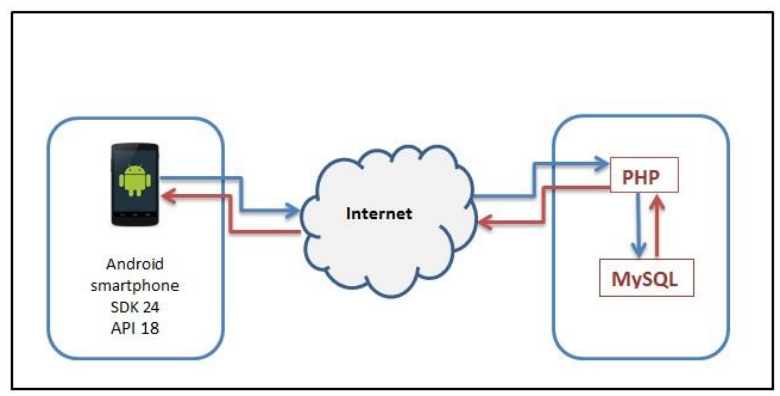

Fig.1 System overview design

\section{B. System activity}

The system consists of activities sequence which led to order the reservation tasks. Every task needs few inputs to proceed to the next task activity as the following:

- The first task is to check the user validation account, or register for new account to transfer to book a flight task.

- At book flight activity the user need to fill the required fields to move to

- Confirm reservation (after check flight availability) which saves the user booking information in the database.

\section{Database design}

The database applied to this application is MySQL which is built with in free host server. Four tables are created which are; users, flight, city, and book. User information, booking information would be saved in users and book table. Admin of system has ability to update flight information by adding new flight, change user information or booking information, contact with user if there is an error or flight cancellationor any an emergency.Figure 2 show database tables and their records.

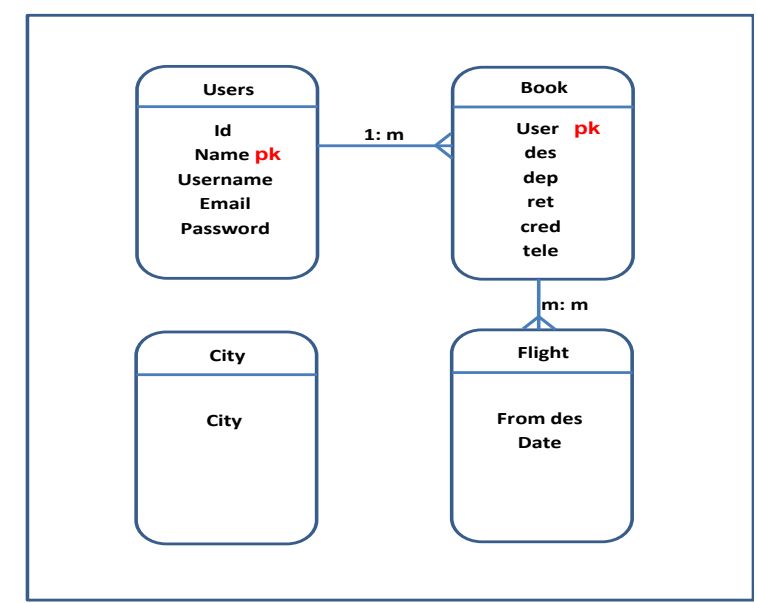

Fig.2 database tables

\section{System implementation}

The application was developed using Android Studio software design. The layout is user friendly designed with $\mathrm{xml}$ and programmed in java classesbythe android studio software. PHP and MySQL in back-end for requests processing, accessing, storing and managing data. Figure 3 represent flowchart of presented system.

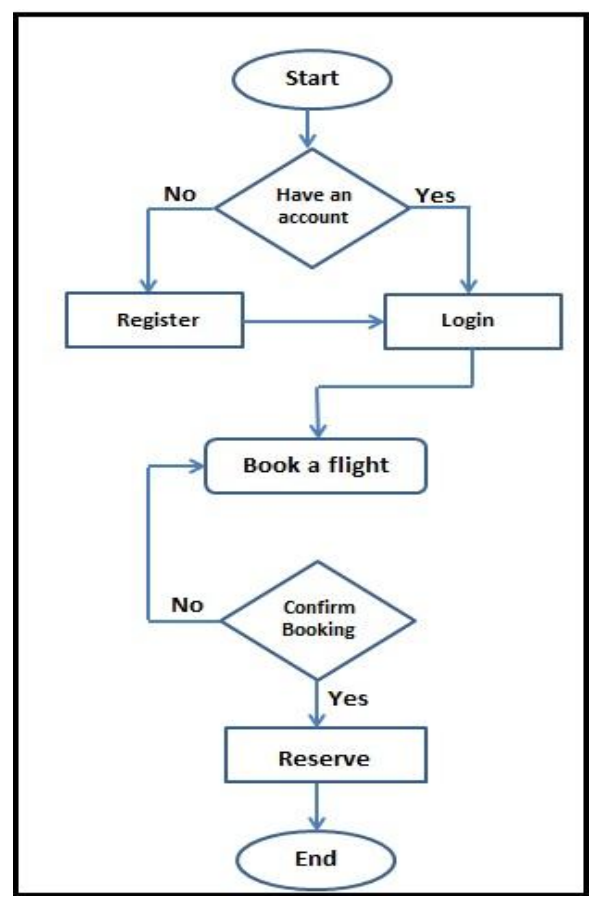

Figure 3 system flowchart

\section{E. System interface}

When client uses the application for the first time he has to register, the next time login entered user name and password will check with the user records in users table in database. In booking flight interface city table associated with spinner tools in flight book activity layout that the user chooses 'from' and 'destination' cities. In the flight 


\section{International Advanced Research Journal in Science, Engineering and Technology \\ ISO 3297:2007 Certified}

Vol. 3, Issue 10, October 2016

table the admin can add new flights available and update airline companies in managing
flight dates. After user book and confirm the flight information and updating fights data. reservation all information will be saved in book table.

A message will appear to user tell him the book is done successfully. Admin of system has ability to update flight information by adding new flight, change user information or booking information, contact with user if there is an error or flight cancellation or any an emergency. Figure 4 show book booking flight user interface.
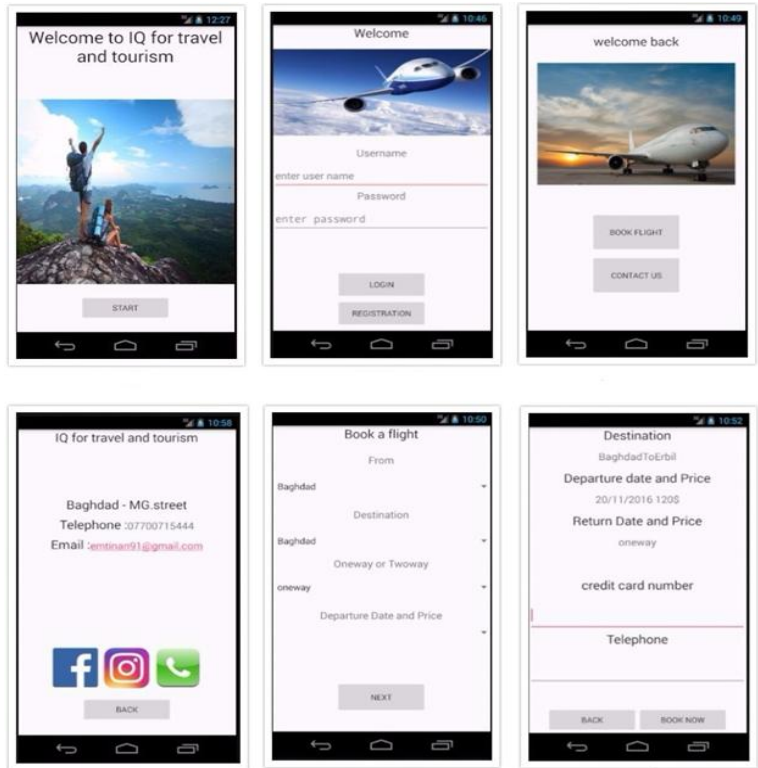

Figure 4 system interface design

\section{F. Class diagram}

As shown in fig 5 class diagram show programmed classes that illustrate system functions occur and their sequence. Start with main activity class of login function which get user information, then moved to menu class to get ready to book flight. In book flight class check if choses of user fight exist in database to book the required one and move to payment class and confirm the reservation and save it in book table in database.

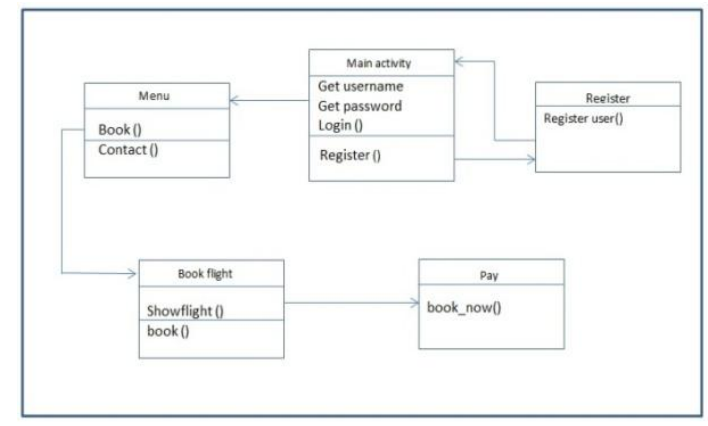

Fig 5 class diagram

\section{CONCLUSION}

In thispaper, we presented an airline reservation system that we design using an android open source program which gives us flexibility in design.This system is useful for passenger in simple the operation of booking and for

\section{ACKNOWLEDGMENT}

I would I would like to thank Dr. Mohammad Najm Abdullah for his supervision during the period of the research, encouragement, comments and help , and thanks all those who stand beside me in my study and give me the encouragement to do this research, Family, and supportive friends.

\section{REFERENCES}

[1] Nojeera malee, 'mobile reservation System for hotel in Malaysia', College of art and science, university Urata Malaysia 2009.

[2] Feichen Shen, 'Situation Aware Mobile Apps Framework', University of Missouri - Kansas City, 2010.

[3] Oyelade 0. J., Fatumo SA, Azeta A.A. and Ayo CK., 'Development of a Mobile Airline Reservation System', Journal of Computer Sciences Its Application, Vol. IS, No. I, June 2009.

[4] Olaniyi, O.M., Ajose, S.Oand Adegoke, M.A. 'Development of a mobile airline reservation and payment system', Int. J. Electronic Finance, Vol. 2010.

[5] Zeyad M. Alfawaer, Mohammad Awni, Saleem Al-Zoubi, 'Mobile E-ticketing reservation system for Amman international stadium in Jordan' INTERNATIONAL JOURNAL Of ACADEMIC RESEARCH, Vol. 3. No.1. January, 2011

[6] Pranjali kharwade, Isha gujarkar, Vidhi Sharma, Shweta Holey, Vaibhavi Datey, Vivek Gupta, 'Smartphone Application for Ticket Reservation and Validation Using Mobile Network', International Journal of Emerging Technology and Advanced Engineering, Vol 4, October 2014.

[7] Ahmed K. Ibrahim and Azman B Ta'a, 'MOBILE - BASED BUS TICKETING SYSTEM IN IRAQ', European Journal of Computer Science and Information Technology, Vol.3, November 2015.

[8] Pooja Gautam, 'ONLINE AIRLINE TICKETING SYSTEM', CENTRIA UNIVERSITY OF APPLIED SCIENCES, September 2015.

[9] Ammar Mohammad Baitalmal, 'Mobile Application Based Parking Reservation System', School of Electrical Engineering and Computer Science, Faculty of Engineering, University of Ottawa, 2015.

[10] Subarnarekha Ghosal, Shalini Chaturvedi, Akshay Taywade and N. Jaisankar, 'Android Application for Ticket Booking and Ticket Checking in Suburban Railways', Indian Journal of Science and Technology, Vol 8(S2), January 2015. Chintan Shah, Wenbin Luo, 'The Design and Implementation of a Workshop Reservation System', American Journal of Engineering Research (AJER), Vol $5,2016$.

[11] Alo O.O., Adeyanju I.A, Muritala A.A., Olabiyisi S.O. and Omidiora E.O., 'Development of a Mobile Airline Reservation Application', Department of Computer Science and Engineering, Ladoke Akintola University of Technology, January 2012.

[12] Richard T. Watson, Pierre Berthon, Leyland F. Pitt, and George M. Zinkhan, 'Electronic Commerce: The Strategic Perspective' by 2008.

[13] IThemes Media, 'Ecommerce for Everybody', 2014.

[14] Syed Hasan, 'DOCUMENTATION OF ONLINE BOOKING SYSTEM', Asia Pacific University of Technology and Innovation, Conference Paper · November 2014.

[15] Randy Connolly, Ricardo Hoar, 'Fundamental of web development', published by Pearson, 2014.

[16] Advance Reservation Through Internet (www.irctc.co.in)

[17] James Gooch, 'Top 8 Benefits of Mobile Ticketing for Mass Transit', masabi, 2016.

[18] ABKDA MOHAMMED ALI AKOUNNI, 'MOBILE-BASED APPLICATION FOR BUS TICKETING SERVICES (MBTS)', COLLEGE OF ARTS AND SCIENCES (CAS), UNIVERSITI UTARA MALAYSIA (UUM), 2009.

[19] Mikko Joutsen, 'MOBILE BANKING AND MOBILE PAYMENTS: CHANGING BANKING SERVICES IN FINLAND', LAPPEENRANTA UNIVERSITY OF TECHNOLOGY, 2013. 